\section{AN EXTENSIVE REVIEW ON: LOW NOISE AMPLIFIER FOR MILLIMETER AND RADIO FREQUENCY WAVES}

\author{
Nitin Agarwala,b, Manish Guptab, Manish Kumarb \\ aR.B.S Engineering Technical Campus Bichpuri Agra, India \\ bECE Department, GLA University Mathura, India
}

Article history

Received

4 February 2021

Received in revised form

30 October 2021

Accepted

9 November 2021

Published Online

20 December 2021

*Corresponding author agarwal_nitin88@rediffmail.com

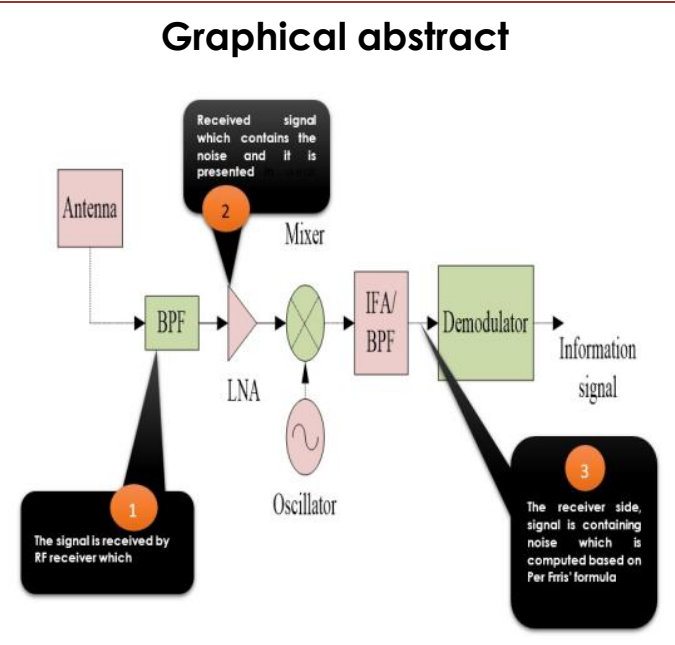

\begin{abstract}
In today's world, radio receiver system is a prevailing wireless technology in that the major part is Low Noise Amplifier (LNA) which widely used to improve weak signals in many applications with millimeter and radio frequency waves such as optical communication, multimode transceivers and measurement instrumentations. The real drawbacks of LNA is that it fails to maintain specific properties in critical conditions like as minimum power consumption, provide low noise figure, input matching and linearity. Additionally, promoted by various application demands, design methods and control methods must require to improve performance of LNA. The performance of LNA can be improved by adding extra components in basic circuit by proper arrangement for millimeter and radio frequency waves. The review paper provides information about design methodology, optimization techniques and control techniques. The different design of LNA is reviewed and analyzed such as 3-stage near-mm Wave LNA, 5-stage near-mm Wave LNA, common-gate amplifier, shunt-feedback amplifier, Resistor-terminated common-source amplifier, Traditional inductor-less amplifiers, cascode connection and double common source. This review paper also provides the information about design circuit diagram. The performance improvement of LNA can be achieved with the help of different techniques and our review based on optimization and control techniques with parameter tuning. Finally, the direction for the future study is presented based on review analysis of LNA.
\end{abstract}

Keywords: LNA, gain, optimization, control techniques, design analysis

(C) 2022 Penerbit UTM Press. All rights reserved

\subsection{INTRODUCTION}

Recently, LNA design has become an inspiring environment, especially for low power applications. A low noise amplifier (LNA) is capable of amplifying the very weak signals receives from the antenna with adding extra noise [1]. LNAs have different advantages, such as low voltage standing ratio (VSWR), minimizing noise figure, high power gain, good stability, and proper impedance matching. LNA can be found in a variety of applications which include Industrial, Scientific, and Medical (ISM) band radios, transponders, mobile phones, GPS reception, and radio wireless local area networks [2]. An LNA is introduced initiated in the RF receiver. Especially in communication systems, it is considered as a basic building block. 
In receiver area of the device, the LNA is an important one because it is serves as an amplifier for electronically amplifying the received signals and it is already placed in front of the receiver [3]. In addition, the important process of the LNA is to increase the low level signal. This amplification process can be analyzed without addition of noise to attain the mandatory signal to noise ratio from the lowest signal level [4]. Similarly, highest signal level condition, the receiver receives the signal and provides amplification first. The basic circuit design of Radio frequency (RF) receiver with LNA is illustrated in Figure 1.

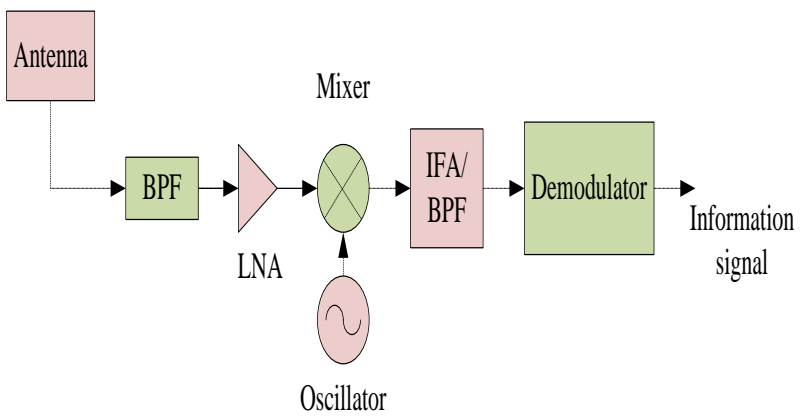

Figure 1 Basic Building Block of RF-LNA [1]

From the Figure 1, at initial condition the signal is received by $\mathrm{RF}$ receiver which sends the signal to band pass filter (BPF). The signal is received with the consideration of antenna system after that which forward to LNA. The LNA get the received signal which contains the noise and it is presented in weak condition [5]. The initial condition of receiver contains few different stages which analysis with formula to compute noise figure (NF). The LNA is a main important electronics device to reduce the noise from received signal by amplifying the signal through increase of gain in signal under subsequent stages [6]. Though, LNA is created itself which inserted into the receiver side signal. Therefore, it is required for an LNA to increase the reference signal power by adding as distortion and low noise as possible, getting required information from received signal by the use of LNA in subsequent stages [7].

\subsection{REVIEW BASED ON MM-WAVE AND RADIO FREQUENCY WAVES}

The LNA can be used for many different applications, including medical, wireless communication systems. The main usage of LNA is the reduction of noise and increasing the low-power signals into high-power signals. In particular, with a lot of radio frequency wave and millimeter-wave (mmWave) applications in the present telecommunication devices due to this wideband circuits have been given much consideration due to their robust versatility and applicability [8]. To meet the applications with $\mathrm{mm}$ Wave and radio frequency waves in the issues of noise behavior and low power signals which should be reduced with the help of LNAThis entry was posted on July 8,2010 . It is able to increase the low power signals into high power signals with reduction of noise [9]. The different design must be developed related on applications in the two different waves of $\mathrm{mm}$ Wave and radio frequency waves. The existing design of LNA amplifier has been analyzed specially in $\mathrm{mm}$ Wave and radio frequency waves with different applications. Some of the research papers related to $\mathrm{mm}$ Wave and radio frequency waves are reviewed in following sections.

\subsection{Design of LNA Amplifier for $\mathrm{mm}$ Wave}

Millimeter wave ( $\mathrm{mm}$ Wave) has recently attracted large research interest in different domain applications especially in communication systems. Since communication systems with $\mathrm{mm}$ Wave, large available bandwidth can potentially lead to rates of multiple Gbps (gigabit per second) per user [10]. The $\mathrm{mm}$ Wave can be utilized in stationary conditions like as backhaul or indoor hotspots which most challenging task to utilize $\mathrm{mm}$ Wave in mobile networks. Difficult condition can be created due to moving condition of receiver and transmitter nodes [11], channels (i.e., structure is complicated) and also coordination between multiple nodes (i.e. complicated) [12].

LNA works on low power signals in mobile network. The $\mathrm{mm}$ Wave can be used in massive multiple input multiple output (MIMO) in communication arrangements [13] for increasing data rate based on availability of wide bandwidth in addition of more no of antennas in small array space. [14] Author designed LNA which enable the best power transfer in receiver signals of antenna. The RF signals are generated with the consideration of photo-detector related to required load impedance within range of frequency; the designed LNA is illustrated in Figure 2.

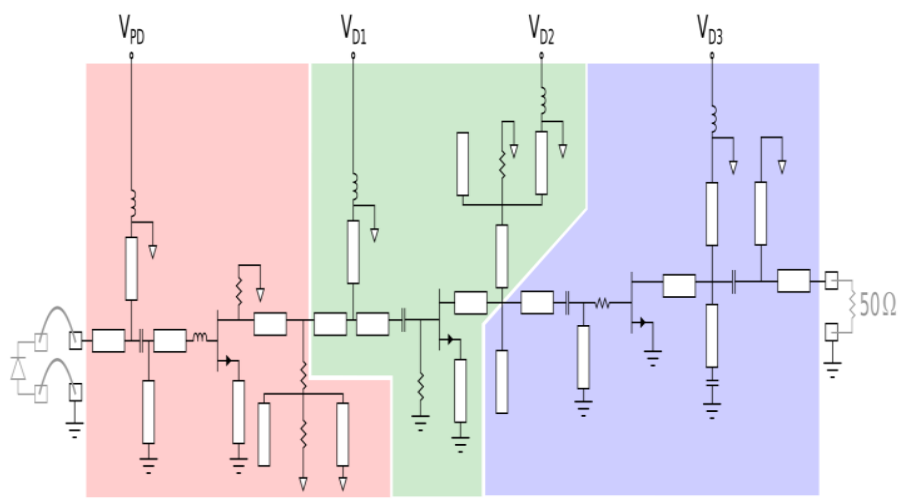

Figure 2 Structure of 3 stages mm Wave LNA [13]

The LNA complete design of the LNA is contains of three amplification stages which can be biasing independently. The LNA can be used to amplify the 
signals in photo-detector. Various methods are developed to give amplification in the output side of photo-detector which is attained with the use of LNA. The three stages LNA have ability towards deliver better optimal power transmission as of the received side signal generated in photo-detector related on load impedance in frequency range [15]. The LNA design with narrowband should allow for a best tradeoff among increasing of gain level and decreasing noise level in received side it works under larger frequency also. When add wire bond inductance on LNA which produce increasing advantages for increasing gain level and decreasing noise level. In the narrowband structure, the LNA operates in best performance which acts as friendly movement by increasing gain compared with conventional amplifiers [16]. The 88.5-110 GHz CMOS based LNA can be designed to meet $\mathrm{mm}$ Wave imaging applications [17]. The developed amplifier consists of five different stages in the cascode topology with Toutput matching and L-type input matching. From five stage of design, the peak signals were distributing the peak gains at initial four stages with two various frequency points. Related on distributing peak gains, LNA attains flat gain response over a wide bandwidth. The LNA performance measurements results provide amplifier features a minimum noise figure is $7.72 \mathrm{~dB}$, $21.5 \mathrm{GHz}$ - 3dB and maximum peak gain $104 \mathrm{GHz}$ 16.7dB. Additionally, LNA has taken a compact core place of $0.05 \mathrm{~mm}^{2}$ and taken $48.6 \mathrm{~mW}$. The pictorial design of LNA amplifier related to $\mathrm{mm}$ Wave imaging applications is presented in Figure 3.

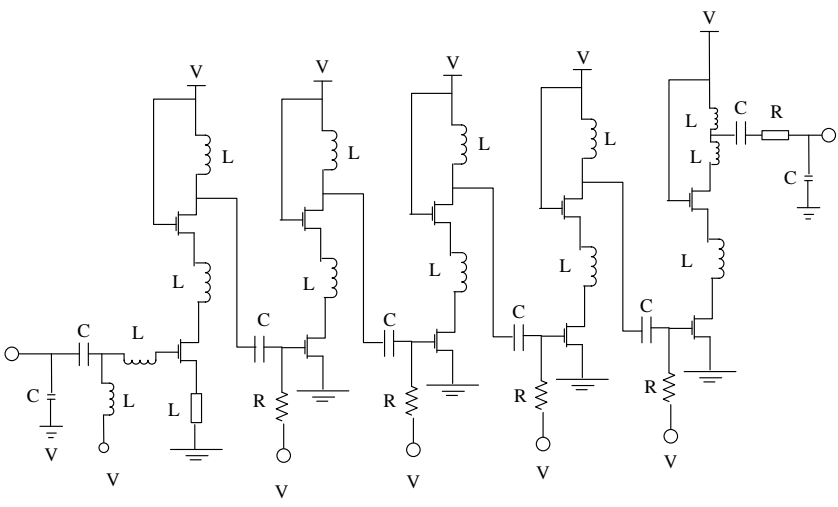

Figure 3 Overall schematic of the 5-stage -mm Wave LNA [17]

The above five stages $\mathrm{mm}$ Wave LNA is mainly used to amplify the low signals into high signals with high gain specially in imaging applications.LNA is mainly connected in receiver side, for high performance $\mathrm{mm}$ Wave imaging receivers which can be mostly important to attain a low resolvable temperature gain. However, with the utilization of the LNA design in RF, high gain and wide bandwidth can be achieved related to small variations. Atiyeh Karimlou et al. [18] have designed inductor less Sub-mW differential Common-Gate Low Noise Amplifier (CGLNA) for ZigBeestandard.The design circuit have the advantages of dual capacitive cross coupling (DCCC) and shunt feedback for reducing power consumption in addition the bandwidth extension capacitors to assits $2.4 \mathrm{GHz}$ ISM band. Different inductor less amplifiers can be designed that provide input impedance matching and voltage gain without any inductors such as common gate amplifier (RCS), shunt feedback amplifier (SFB) and resistor terminated common source (R-CS) amplifier. Based on the conventional methods, different LNA can be designed to meet required voltage gain in mmWave applications which designed in single or combination.

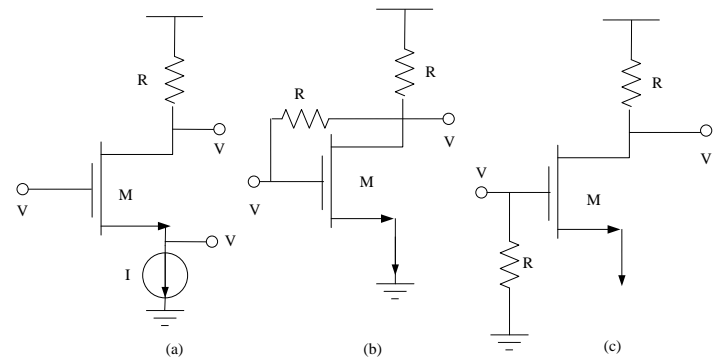

Figure 4 Traditional inductor-less amplifiers: (a) common-gate amplifier, (b) shunt-feedback amplifier and (c) Resistorterminated common-source amplifier [19]

LNA can be a essential building block in the receiver for increaing gain, must qualify best impedance matching and reduced noise figure related to freqency band. To achieve better performance in mmWave applications, best LNA design sholud essential. The LNA should be design to meet $\mathrm{mm}$ Wave applications some of the designs are discussed in this section. The radio frequency wave applications with LNA releted analysis presented in below section

\subsection{Design of LNA Amplifier for Radio Frequency}

The radio frequency wave LNA, which is essential to attain best performance from low signals [19]. For example, wireless LANs, Wi-Fi, cell phones and radios astronomy were cover frequency wave which presented in $2.4 \mathrm{GHz}$. In the receiver side, LNA block can be one of the most important block in all devices. Because, the signal come from an antenna are moderately, weak such as little micro voltage $(\mu \mathrm{V})$ and nano voltage $(\mathrm{nV})$ range of the signal. This nano and micro signals should be amplified which attained with the utilization of LNA to increase signal strength. To maintain input-output matching network, the special LNA design is required. The authors [20] have designed LNA to achieve high gain in addition low noise figure which presented in Figure 5.

The LNA design can be developed based on cascode connection. The circuit diagram consists of four different stages such as input impedance matching stage, common source stage (CS), common gate stage (CG) and the output impedance matching stage. Initial stage of designed LNA is input impedance network consists of inductors, capacitors 
and resistances. The designed LNA can achieve important properties such as less consumption power, effortless input output matching, high gain and less noise figure. The author [21] have designed CMOS based LNA to meet specific properties in radio frequency wave receiver side. The developed LNA was designed with double common source structure that design solve narrowband problem and attain wide band input matching in band of $3-12 \mathrm{GHz}$ which presented in Figure 6.

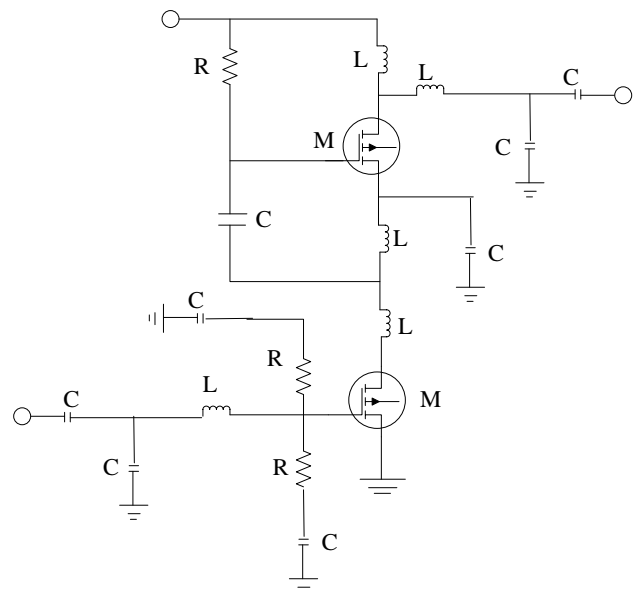

Figure 5 Circuit structure of the developed LNA Circuit [20]

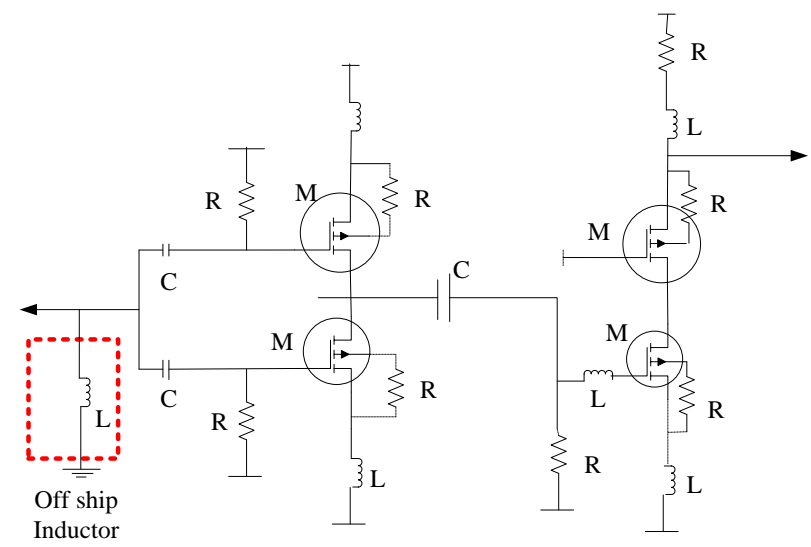

Figure 6 Circuit diagram of the LNA Circuit with double common source [21]

The common source design is a narrow band structure here the double common source structure is utilized in input and two frequency points. These parameters were created to cover whole frequency band with resonating parasitic inductors and capacitances which located in the source terminals. To reduce noise figure of the receiver, the series resistors are introduced in the circuit which connected to the source bulk substrate resistance. The second stage of the circuit is utilized to attain low power structure and high gain. The authors [22] have designed T-type matching based LNA design to meet requirements especially in radio frequency waves. Sometimes, T-type matching circuit with passive components are used in the input matching circuit which attained matching input but required gain cannot achieved and power consumption also high. Farshad Eshghabadi et al. [23] have designed radio frequency CMOS based LNA. This design aims to attain high gain through first pass silicon fabrication success to reduce cost in addition time overriding progress. Saeed Ghaneei Aarani et al. [24] have designed ultra wide band (UWB) LNA which used to increment in noise figure in addition gain improvement using 0.18 um radio frequency CMOS technology. This structure was designed with two cross coupled capacitors (CCC) in addition new gain flattening method with cascode common gate design. The LNA design should require control techniques to meet better performances of high gain, low noise figure and less consumption power.

\subsection{CONTROL TECHNIQUES OF LNA}

LNA design has been increased in multi band transceiver to meet different applications with better performance. The LNA should have specific characteristics of low noise figure, high gain, and supply best input matching with low ingestion of power. There are various LNA design presented to attain more linear that working related on IM3 cancelation utilizing piecewise linear, feed-forward, feedback, low impedance termination in addition prepost distortion methods. The different control techniques are essential to meet all requirements with better and high gain attainments. The control techniques are reviewed and listed below.

\subsection{Optimization based Control Techniques}

With the improvement of applications in various domains, such as information sharing, industrial settings, healthcare, and agriculture, Generally, these presentations mostly involve difficulties with a front-end circuit that contains an LNA to deliver the signals with high gain and low power consumption. The LNA must work continuously to provide proper signals in specific applications. To ensure the performance of the LNA, control techniques are included in the design operation of the LNA, which is attained with the help of an optimization algorithm. Manish Kumar et al. [25] have designed an LNA for wide band applications using the particle swarm optimization (PSO) technique. This article provides the design investigation of low voltage and low power enhancement in LNA intended for wide band presentations. Based on design experiments with LV and LP, a biasing metric was developed for analogue circuits with radio frequency. The designed LNA contains two different phases, such as the current reuse topology and the mutually coupled CS phase. The first phase was a grouping of the top NMOS transistors and PMOS transistors with consideration of sequence inductive peaking in the feedback circle. From the LNA design, 
passive component parameters can be optimised with the help of the PSO technique.

Chabungbam Lison Singh et al. [26] have introduced Particle Swarm Optimization with an Aging Leader and challengers (ALCPSO)-based control strategy in LNA. It was mainly used to CMOS analog amplifier with low noise intended for automate sizing. The sizing was achieved in LNA with the help of optimization algorithm. Charles baylis et al. [27] has developed Pareto optimization to improve design parameters in LNA. Here, fundamental solution methods for the source reflection coefficient supplying the high gain under a constraint of noise figure and also low gain under a constraint of noise figure. The developed method was working related to small signal $S$ parameters and noise parameters of the device. YiTsen Ku et al. [31] have developed gain boosted and noise optimization technique in special design CMOS wide band low voltage LNA. The designed LNA have gain boosted technique with negative feedback condition for increasing high frequency gain towards wide bandwidth and attain high gain. Additionally, it has been utilized mirror bias technique to attain complete output swing. Because of this technique, this operating current cannot change due to supply voltage variation.

Ram Kumar et al. [32] have introduced multi firefly algorithm to optimize the parameters of $5.5 \mathrm{GHz}$ LNA. The firefly algorithm was utilized to improve the specific values of LNA like as output and input matching, gain noise figure in addition linearity by satisfying complete constraints. Here, five parameters were considered to optimize which taken as multi objective function. Weighed sum technique was utilized to change multi objective function to single objective function. The design LNA has the inductive source degeneration topology with cascode structure. Kosuke Katayama et al. [30] have presented Bias-Optimization Technique for reducing the number of measurements in LNA. This technique was utilized to reconstructing a completed model of LNA amplifier by reducing number of measurements solutions. Here, design an eight stage D-band LNA with the utilization $65 \mathrm{~nm}$ CMOS method. This method was mainly used to increase gain of amplifier. Ram Kumar et al. [32] have presented firefly algorithm to optimize LNA design parameters. The main parameter of LNA was optimized such as gain and noise figure. Here, two main objective functions were concentrated such as maximizing gain and minimizing NF. These two parameters were considered as the single objective function. To handle the constraints of LNA, the penalty factor method was initialized. Additional parameters also considered in this case such as stability, linearity and power consumption. The designed LNA have different structure such as cascode connection with inductive source degeneration topology. The LNA was designed for $5.5 \mathrm{GHz}$ frequency. Chun-Chieh Chen et al. [28] have presented gain and product optimization technique for increasing gain in LNA. The design was presented cascode LNA, common gate and dual bandwidth that decreasing value of capacitance in equivalent with the consideration the resonator loads. The optimization algorithm was utilized to select best bandwidth in addition gain product of LNA. Finally, the input impedance and noise factor of the designed LNA was examined.

Angelos Papadimitriou et al. [33] have developed genetic computation to optimal parameters of radio frequency with LNA. This article was introducing a methodology for multi objective with optimization using an analytical model of LNA in MOS transistor related with genetic computation. The best performance was derived by a figure of merit (FoM) which considers both RF performance and power efficiency of LNA. Here, short channel model was utilized to analysis the FoM model which presents MOS inversion level present in the moderate inversion. $R$. Tamilchelvan et al. [34] have introduced nonlinear technique to improve performance of wideband microwave amplifier design adopting negative image synthesis. The different microwave properties and RF properties of the amplifier were analysis and investigated. The developed method was amalgamated nonlinear model of the transistor in LNA which considered as input. Additionally, negative image synthesis intended for impedance matching systems at the output intended for input phase of the amplifier. The designed LNA parameters of inductor, capacitor and chip resistor were selected with the help of nonlinear technique. From this analysis, optimization methods were used to enhance the performance of LNA design in receiver side for increasing gain and reducing power consumption so on. To attain best performance of LNA, various control techniques are available which reviewed in below section.

\subsection{Different Control Techniques}

Generally, an electrical system can be combined with various components. Due to changes in current and voltage, noise is generated in circuits. Very low signals are amplified with the help of LNA. The noise of the system can be reduced and gain can be increased with LNA. Mostly LNA is used in receiver side.

The LNA's design is based on the $S$ parameter in the transistor component. The LNA should have different properties such as good dynamic range, good linearity, good sensitivity is desirable, third order intercept point, amplifier stability. with the selection of the proper transistor, and proper biasing conditions, bandwidth, gain, and noise figure can be improved. Different control techniques are available to increase gain, bandwidth, and reduce noise in LNA designs. The various control techniques of LNA are reviewed in this section.

Abolfazl Zokaei et al. [35] have designed 65nm linear broad band differential LNA with the help of post distortion method. The post distortion method was utilized to a wide band differential common gate LNA which used with noise cancelation technique. The main objective of the technique was noise channel cancellation of the CG input transistor under various 
output nodes by increasing gain of input signal. From the input transistor, noise channel can be passes by two various paths with equal polarity which removed at the differential output modes. Mohsen Hayatiet al. [36] has presented current reused technique for noise cancelling in UWB-LNA. In the UWB LNA amplifier, a combination of current reused and noise cancelling technique for reducing noise, power consumption and increasing gain, linearity. With the utilization of resistive feedback methods in addition of inductive source degeneration, the best input impedance matching was attained.

Yu-Teng Chang et al. [37] have introduced current reused methods intended for stable matching in addition maintains linearity in CMOS based variable gain low noise amplifier (VGLNA). The developed VRLNA designs have three amplifier phases' in addition three digital gain control bits. Initial stage was a common source amplifier. Second in addition of third stage were reused techniques. To protect control gain in addition dc power, current reuse method in addition connect the digital switch among lower and upper transistors. Because of constant dc current, the reused stage of input impedance was stable. The output impedance was stabilized with connection of ac grounding capacitor at source terminal of the upper transistor under gain switching. Yo-Sheng Lin et al. [38] have presented $\lambda / 2$-spiral-inductor-basedpositive feedback technique in low power and wideband LNA. The developed technique was used at the input common source stage for increasing gain value. Additionally, T-match method was used to attain output impedance matching, input impedance matching and noise figure.

Kai Jing et al. [39] have presented asymmetric $\pi$ filter and shunt-peaking technique for increasing gain and input with output matching in LNA. Shunt feedback method was used to improve wide operating point as well as form using noise reduction methods. Jianquan $\mathrm{Hu}$ et al. [40] have presented Multiple Bandwidth Extension Techniques in broadband LNA. With the utilization of multiple bandwidth extension methods, consisting double series peaking techniques, shunt and feedback methods, the designed LNA attains a calculated average gain of $26.5 \mathrm{~dB}$ from one to $40 \mathrm{GHz}$ with reduced amount of noise figure.

Dalia Elsheakh et al. [41] have introduced Differential Noise Canceling method in UWB LNA. The developed system contains of a CMOS differential LNA and off chip micro strip antenna with the consideration of differential noise canceling (DNC) method. Here, design antenna was printed on a low cost FR $\$$ substrate and trapezoidal dipole shaped with balun in addition dimensions $10 \times 10 \times 0.8 \mathrm{~mm}^{3}$. To improve antenna impedance matching, balun circuit can be integrated with the antenna in ground condition. To remove noise from LNA, differential block was initialized. Shita Guo et al. [42] have presented Boosting Technique to increase gain in LNA and noise reduction to decrease noise in LNA. A transformer feedback boosting method was developed in single ended cascode design towards reduce noise and increase gain. The developed method was theoretically calculated and complete analysis was presented. To increase gain and reduce noise of LNA, the presented method was used and analyzed. Guangy in Feng et al. [43] have presented Bandwidth Extension Technique to improve gain and decreasing noise from three stage cascode LNA. The three stages cascode LNA was designed related to negative drain source transformer and pole converging technique.

\subsection{PERFORMANCE ANALYSIS}

LNA design performance can be improved with the help of control techniques by increasing gain and reducing noise. Optimization algorithms were utilized to increase gain and reducing noise by select optimal parameters in LNA design. The different optimization based control technique in LNA amplifier is listed in Table 1.

Table 1 LNA control techniques with optimization

\begin{tabular}{|c|c|c|c|c|}
\hline $\begin{array}{l}\text { S. } \\
\text { No. }\end{array}$ & Author & Year & Technique & Design \\
\hline 1 & $\begin{array}{c}\text { Manish Kumar } \\
{[25]}\end{array}$ & 2019 & $\begin{array}{c}\text { Particle } \\
\text { Swarm } \\
\text { Optimization }\end{array}$ & $\begin{array}{c}\text { Low } \\
\text { power, low } \\
\text { voltage } \\
\text { LNA }\end{array}$ \\
\hline 2 & $\begin{array}{c}\text { Chabungbam } \\
\text { Lison Singh } \\
{[26]}\end{array}$ & 2017 & $\begin{array}{c}\text { Particle } \\
\text { Swarm } \\
\text { Optimization } \\
\text { with an } \\
\text { Aging } \\
\text { Leader }\end{array}$ & $\begin{array}{l}\text { low-noise } \\
\text { CMOS }\end{array}$ \\
\hline 3 & $\begin{array}{c}\text { Charles Baylis } \\
\text { [27] }\end{array}$ & 2015 & $\begin{array}{c}\text { Pareto } \\
\text { optimization }\end{array}$ & $\begin{array}{l}\text { low-noise } \\
\text { amplifier }\end{array}$ \\
\hline 4 & $\begin{array}{c}\text { Chun-Chieh } \\
\text { Chen [28] }\end{array}$ & 2016 & $\begin{array}{c}\text { Product } \\
\text { Optimization } \\
\text { Technique }\end{array}$ & $\begin{array}{c}\text { Dual- } \\
\text { Wideband } \\
\text { CMOS LNA }\end{array}$ \\
\hline 5 & $\begin{array}{c}\text { Deepak Joshi } \\
{[29]}\end{array}$ & 2017 & $\begin{array}{c}\text { Hybrid } \\
\text { Particle } \\
\text { Swarm } \\
\text { Optimization } \\
\text { with Levy } \\
\text { Flight } \\
\end{array}$ & $\begin{array}{c}2.4 \mathrm{GHz} \\
\text { CMOS LNA }\end{array}$ \\
\hline 6 & $\begin{array}{c}\text { Kosuke } \\
\text { Katayama } \\
{[30]}\end{array}$ & 2016 & $\begin{array}{c}\text { Bias- } \\
\text { Optimization } \\
\text { Technique }\end{array}$ & $\begin{array}{c}\text { 14.4-dB } \\
\text { CMOS D- } \\
\text { band LNA }\end{array}$ \\
\hline 7 & Yi-Tsen Ku [31] & 2018 & $\begin{array}{c}\text { Gain } \\
\text { Boosted and } \\
\text { Noise } \\
\text { Optimized } \\
\text { Techniques } \\
\end{array}$ & $\begin{array}{c}\text { CMOS } \\
\text { wide-band } \\
\text { low- } \\
\text { voltage } \\
\text { LNA }\end{array}$ \\
\hline 8 & $\begin{array}{c}\text { Ram Kumar } \\
\text { [32] }\end{array}$ & 2016 & $\begin{array}{c}\text { firefly } \\
\text { algorithm }\end{array}$ & $\begin{array}{c}5.5-\mathrm{GHz} \\
\text { CMOS LNA }\end{array}$ \\
\hline 9 & $\begin{array}{c}\text { Angelos } \\
\text { Papadimitriou } \\
{[33]}\end{array}$ & 2017 & $\begin{array}{c}\text { Genetic } \\
\text { Computation }\end{array}$ & RF- LNA \\
\hline 10 & $\begin{array}{l}\text { R. } \\
\text { Tamilchelvan } \\
{[34]}\end{array}$ & 2018 & $\begin{array}{l}\text { Nonlinear } \\
\text { Technique }\end{array}$ & $\begin{array}{c}\text { Wideband } \\
\text { Microwave } \\
\text { Amplifier }\end{array}$ \\
\hline
\end{tabular}


Table 2 LNA control techniques with parameters

\begin{tabular}{|c|c|c|c|c|c|c|c|c|c|}
\hline S. No & Author & B.W & Gain & NF & IIP3 & FoM & $\begin{array}{c}\text { Power } \\
(\mathbf{n W})\end{array}$ & Tech & Size(mm2) \\
\hline 1 & A. Saberkari [44] & $3.1-10.6$ & 12.1 & 4.56 & -12.5 & 1.83 & 13.6 & $180 \mathrm{~nm}$ & - \\
\hline 2 & G. Gramegna [45] & $2.8-7.5$ & 9 & 3.6 & -10.5 & - & 3.8 & $180 \mathrm{~nm}$ & 0.77 \\
\hline 3 & K.V. Reddy [46] & $1-10.3$ & 16 & 4.1 & 11 & - & 10.9 & $180 \mathrm{~nm}$ & 0.2 \\
\hline 4 & A. Sahafi [47] & $2.35-9.37$ & 10.3 & 3.68 & -4 & - & 9.97 & $130 \mathrm{~nm}$ & 0.39 \\
\hline 5 & S. Arshad [48] & $3.2-10.64$ & 17 & 2.5 & - & - & 16.5 & $180 \mathrm{~nm}$ & 0.56 \\
\hline 6 & A. Taibi [49] & $3-10$ & 16 & 3.7 & - & - & 16.2 & $65 \mathrm{~nm}$ & 0.12 \\
\hline 7 & M. Sun [50] & $3-12$ & 13.5 & 4.3 & -7 & 4.33 & 8.5 & $130 \mathrm{~nm}$ & 0.85 \\
\hline 8 & \begin{tabular}{c} 
Benqing Guo [51] \\
\hline 9
\end{tabular} & $0.1-2$ & 13 & 2.9 & 10.6 & - & 21.3 & $\begin{array}{c}\mathrm{CMOS} \\
0.18^{\mu} \mathrm{m}\end{array}$ & 0.63 \\
\hline 10 & $\begin{array}{c}\text { Mahdi Parvizi [52] } \\
\text { Taeyoung Chung } \\
\text { [53] }\end{array}$ & $0.1-2.2$ & 17.5 & 4.9 & -11.5 & 17.9 & 0.4 & $\begin{array}{c}\mathrm{CMOS} \\
0.13 \mu_{\mathrm{m}}\end{array}$ & 0.0052 \\
\hline 11 & \begin{tabular}{c} 
Hankyu Lee [54] \\
\hline 12
\end{tabular} & $0.1-1.6$ & 12.3 & 2.1 & 5.5 & - & 20.08 & $\begin{array}{c}\mathrm{CMOS} 65 \\
\mathrm{~nm}\end{array}$ & 0.014 \\
\hline & Lanqi Liu [55] & $0.01-1.7$ & 13 & 1.7 & 0 & 14.6 & 13 & $\begin{array}{c}\mathrm{CMOS} 65 \\
\mathrm{~nm}\end{array}$ & 0.008 \\
\hline
\end{tabular}

\subsection{FINDINGS}

In order to reduce noise and increasing gain in electrical and electronic devices by LNA are the most appreciated technology in the wireless communication networks. Hence, importance of increasing gains in low signals of devices in wireless devices. The low signals are changed into high signals for increasing gain by LNA with proper controlling technique and optimization algorithm. The optimal design parameters of the LNA are selected with the help of optimization algorithm. Additionally, the controller techniques are used to adjust the design parameters in LNA design for reducing noise cancel and increasing gain also. So many research works have been proposed for the proper controller in LNA, some of the reviews are presented in previous sections. From the review we are suggesting that the optimization or control techniques are highly essential to increasing gain and reducing noise from the circuit. The future control techniques or optimization algorithms in LNA will be used to improve the performance of circuits. The design parameters of LNA will be adjusted and optimized with the help of control techniques. In future, different novel techniques will be introduced to improve the performance of LNA by adjusting design parameters.

The various control techniques are used to increase gain and reducing noise from LNA design which constraints are presented in Table 2. The specific parameters of bandwidth, noise figure, power, technology and sizes were analyzed.

\subsection{CONCLUSION}

In this paper, LNA design and control techniques were reviewed to improving gain and reducing noise in different applications with millimeter and radio frequency waves. The LNA is a most important part in radio receiver system in wireless devices with millimeter and radio frequency waves. The review is progressed based on different techniques to improve gain and reducing noise in LNA. The different design of LNA is reviewed such as 3-stage near-mm Wave low noise amplifier, 5-stage near-mm Wave LNA, common-gate amplifier, shunt-feedback amplifier, Resistor-terminated common-source amplifier, and traditional inductor less amplifiers, cascode connection and double common source. The notable optimization and control techniques with parameters were analyzed and reviewed in this paper. The comparative analysis of control techniques and optimization techniques were analyzed and tabulated. The future direction also provided in this paper for increasing gain and reducing noise in LNA with proper controlling and optimization techniques. In the future, cascaded feedback techniques need to be initialized for improving performance of the LNA in system to meet different applications especially in wireless communication system.

\section{References}

[1] Xue Jun Li and Yue Ping Zhang. 2018. CMOS Low Noise Amplifier Design for Microwave and $\mathrm{mm}$ Wave Applications. Progress in Electromagnetics Research. 161: 57-85.

[2] Filiz Güneş, Salih Demirel, and Selahattin Nesil. 2016. Design Optimization of LNAs and Reflectarray Antennas using the Full-wave Simulation-based Artificial Intelligence Models with the Novel Metaheuristic Algorithms. Simulation-Driven Modeling and Optimization. Springer, Cham. 261-298.

[3] Jennifer Zaini-Desevedavy, Frédéric Hameau, Thierry Taris, Dominique Morche, and Patrick Audebert. 2018. An ultralow Power $28 \mathrm{~nm}$ FD-SOI Low Noise Amplifier based on Channel Aware Receiver System Analysis. Journal of Low Power Electronics and Applications. 8(2): 10. 
[4] Yao Yao, Dongxu Yue, and Kaiqi Wan. 2018. A Ku-band Wideband Low Noise Amplifier. 4th Workshop on Advanced Research and Technology in Industry (WARTIA 2018). Atlantis Press.

[5] Peter Sarson, Tomonori Yanagida, and Kosuke Machida. 2018. Measuring Group Delay of Frequency Down converter Devices Using a Chirped RF Modulated Signal. Journal of Electronic Testing. 34(3): 233-253.

[6] Mohammad Reza Nikbakhsh, Ebrahim Abiri, Hossein Ghasemian, and Mohammad Reza Salehi. 2018. A Two Stage Variable-gain Low-noise Amplifier for X-Band in 0.18 áÁm CMOS. Wireless Personal Communications. 98(1): 173-187.

[7] Prashanth, Chittireddy, and Mahesh Mudavath. 2016. CMOS Low Noise Amplifier for IEEE 802.11 b Wireless LAN Applications. 9(2).

[8] Haohong Yu, Yong Chen, Chirn Chye Boon, Chenyang Li, Pui-In Mak, and Rui P. Martins. 2018. A 0.044-mm 2 0.5-to-7$\mathrm{GHz}$ Resistor-plus-source-follower-feedback Noisecancelling LNA Achieving a Flat NF of $3.3 \pm 0.45 \mathrm{~dB}$. IEEE Transactions on Circuits and Systems II: Express Briefs 66. 1: 71-75.

[9] Pierre Payet, M. Guery, Jeremy Raoult, and Laurent Chusseau. 2017. Out-of-band Disturbance of mm-wave EMI on RF Front-ends. Microelectronics Reliability. 76: 670673.

[10] Russell Ford, Menglei Zhang, Marco Mezzavilla, Sourjya Dutta, Sundeep Rangan, and Michele Zorzi. 2017. Achieving Ultra-low Latency in 5G Millimeter Wave Cellular Networks. IEEE Communications Magazine. 55(3): 196-203.

[11] Ming Xiao, Shahid Mumtaz, Yongming Huang, Linglong Dai, Yonghui Li, Michail Matthaiou, George K. Karagiannidis et al. 2017. Millimeter Wave Communications for Future Mobile Networks. IEEE Journa on Selected Areas in Communications. 35(9): 1909-1935.

[12] R. W. Heath, N. Gonzalez-Prelcic, S. Rangan, W. Roh, and A. Sayeed. 2016. An Overview of Signal Processing Techniques for Millimeter Wave MIMO Systems. IEEE J. Sel. Top. Signal Process. 10(3): 436-453.

[13] Jinseok Choi, Gilwon Lee, Ahmed Alkhateeb, Alan Gatherer, Naofal Al-Dhahir, and Brian L. Evans. 2020. Advanced Receiver Architectures for Millimeter Wave Communications with Low-Resolution ADCs. arXiv preprint arXiv:2003.03683.

[14] Laurens Bogaert, Haolin Li, Kasper Van Gasse, Joris Van Kerrebrouck, Johan Bauwelinck, Gunther Roelkens, and Guy Torfs. 2020. 36 Gb/s Narrowband Photoreceiver for mmWave Analog Radio-over-Fiber. Journal of Light Wave Technology

[15] Fabian Thome, Arnulf Leuther, and Oliver Ambacher. 2020. Low-Loss Millimeter-Wave SPDT Switch MMICs in a Metamorphic HEMT Technology. IEEE Microwave and Wireless Components Letters. 30(2): 197-200.

[16] Karkare, S., P. S. Barry, C. M. Bradford, S. Chapman, S. Doyle, J. Glenn, S. Gordon et al. 2020. Full-Array Noise Performance of Deployment-Grade SuperSpec mm-Wave On-Chip Spectrometers. Journal of Low Temperature Physics. 2020: 1-9

[17] Guangyin Feng, Chirn Chye Boon, Fanyi Meng, Xiang Yi, and Chenyang Li. 2016. An 88.5-110 GHz CMOS Low-noise Amplifier for Millimeter-wave Imaging Applications. IEEE Microwave and Wireless Components Letters. 26(2): 134136.

[18] Atiyeh Karimlou, Roya Jafarnejad, and Jafar Sobhi. 2016. An Inductor-less sub-mW Low Noise Amplifier for Wireless Sensor Network Applications. Integration. 52: 316-322.

[19] Lee, M., Kwon, I. 2018. 3-10 GHz Noise-cancelling CMOS LNA using gm-boosting Technique. IET Circ Dev Syst. 12(1): 12-6

[20] Mahesh Mudavath, K. Hari Kishore, Azham Hussain, and C. S. Boopathi. 2020. Design and Analysis of CMOS RF Receiver Front-End of LNA for Wireless Applications. Microprocessors and Microsystems. 2020: 102999.
[21] Farzad Daryabari, Abdulhamid Zahedi, Abbas Rezaei, and Mohsen Hayati. 2019. Gain-controlled Noise-reduction LNA Design using Source-bulk Resistors and Double Common-source Topology. Integration. 68: 50-61.

[22] B. Sene, A. Werthof, V. Issakov, A. 2017. 30-to-110 GHz Broadband LNA using T-type Matching and Admittance Inverter in $0.35 \mu \mathrm{m}$ SiGe:C Technology. IEEE International Conference on Microwaves, Antennas, Communications and Electronic Systems (COMCAS), Tel-Aviv. 1-4

[23] Farshad Eshghabadi, Fatemeh Banitorfian, Norlaili Mohd Noh, Mohd Tafir Mustaffa, and Asrulnizam Abd Manaf. 2016. Post-process Die-level Electromagnetic Field Analysis on Microwave CMOS Low-noise Amplifier for First-pass Silicon Fabrication Success. Integration. 52: 217-227.

[24] Saeed Ghaneei Aarani, Mohsen Hayati, and Amir Hossein Kazemi. 2019. A Novel UWB Low-noise Amplifier Design using Double Capacitor Cross-coupled Feedback. AEUInternational Journal of Electronics and Communications. 104: 1-9.

[25] Manish Kumar and Vinay Kumar Deolia. 2019. Performance Analysis of Low Power LNA using Particle Swarm Optimization for Wide Band Application. AEUInternational Journal of Electronics and Communications. 111: 152897.

[26] Chabungbam Lison Singh, Chabungbam Anandini, Ashim Jyoti Gogoi, and K. L. Baishnab. 2018. Automated Sizing of Low-noise CMOS Analog Amplifier using ALCPSO Optimization Algorithm. Journal of Information and Optimization Sciences. 39(1): 99-111.

[27] Charles, Robert J. Baylis, Marks, and Lawrence Cohen. 2016. Pareto Optimization of Radar Receiver Low-noise Amplifier Source Impedance for Low Noise and High Gain. International Journal of Microwave and Wireless Technologies. 8(8): 1133-1139.

[28] Chun-Chieh Chen, and Yen-Chun Wang. 2017. A Dualwideband CMOS LNA using Gain-bandwidth Product Optimization Technique. Circuits, Systems, and Signal Processing. 36(2): 495-510.

[29] Deepak Joshi, Satyabrata Dash, Ayush Malhotra, Pulimi Venkata Sai, Rahul Das, Dikshit Sharma, and Gaurav Trivedi. 2017. Optimization of $2.4 \mathrm{GHz}$ CMOS Low Noise Amplifier using Hybrid Particle Swarm Optimization with Lévy Flight. 30th International Conference on VLSI Design and 2017 16th International Conference on Embedded Systems (VLSID). IEEE, 2017. 181-186.

[30] Kosuke Katayama, Kyoya Takano, Shuhei Amakawa, Takeshi Yoshida, and Minoru Fujishima. 2016. 14.4-dB CMOS D-band Low-noise Amplifier with 22.6-mW Power Consumption Utilizing Bias-optimization Technique. 2016 IEEE International Symposium on Radio-Frequency Integration Technology (RFIT). IEEE, 2016. 1-3.

[31] Yi-Tsen Ku and San-Fu Wang. 2018. A New Wide-band Low-voltage Low-noise Amplifier with Gain Boosted and Noise Optimized Techniques. IETE Journal of Research. 1-7.

[32] Ram Kumar, Abhishek Rajan, Fazal A. Talukdar, Nilanjan Dey, V. Santhi, and Valentina E. Balas. 2017. Optimization of $5.5-\mathrm{GHz}$ CMOS LNA Parameters using Firefly Algorithm. Neural Computing and Applications. 28(12): 3765-3779.

[33] Angelos Papadimitriou and Matthias Bucher. 2017. Multiobjective Low-noise Amplifier Optimization using Analytical Model and Genetic Computation. Circuits, Systems, and Signal Processing. 36(12): 4963-4993.

[34] R Tamilchelvan, and V. Jawahar Senthilkumar. 2018. Design and Optimization of Wideband Microwave Amplifier Using Nonlinear Technique. Wireless Personal Communications. 99(4): 1589-1604.

[35] Abolfazl Zokaei, and Amir Amirabadi. 2018. A 65 nm Linear Broad-band Differential Low Noise Amplifier Using Post Distortion Technique. Microelectronics Journal. 74: 24-33.

[36] Mohsen Hayati, Sajad Cheraghaliei, and Sepehr Zarghami. 2018. Design of UWB Low Noise Amplifier using Noise-canceling and Current-reused Techniques. Integration. 60: 232-239. 
[37] Yu-Teng Chang, and Hsin-Chia Lu. 2019. A \$ $\vee \$$-Band Low-Power Digital Variable-gain Low-noise Amplifier using Current-reused Technique with Stable Matching and Maintained OPIdB. IEEE Transactions on Microwave Theory and Techniques. 67(11): 4404-4417.

[38] Yo-Sheng Lin, and Kai-Siang Lan. 2019. W-band Low-noise Amplifier using $\lambda / 2$-spiral-inductor-based Positive Feedback Technique in $90 \mathrm{~nm}$ CMOS. Analog Integrated Circuits and Signal Processing. 99(3): 679-691.

[39] Kai Jing, Ningmei Yu, and Xing Quan. 2019. An S-to Ku-wideband Low-noise Amplifier using Asymmetric $\pi$ Filter and Shunt-peaking Technique with Simultaneous Input Matches and Noise Flatness. Microwave and Optical Technology Letters. 61 (6): 1509-1516.

[40] Jianquan Hu, and Kaixue Ma. 2019. A 1-40-GHz LNA MMIC using Multiple Bandwidth Extension Techniques. IEEE Microwave and Wireless Components Letters. 29(5): 336338.

[41] Dalia Elsheakh, Heba Shawkey, and Sherif Saleh. 2019. A 9-10.6 GHz Microstrip Antenna-UWB Low Noise Amplifier with Differential Noise Canceling Technique for loT Applications. International Journal of Communications, Network and System Sciences. 12(11): 189-197.

[42] Shita Guo, Tianzuo Xi, Ping Gui, Daquan Huang, Yanli Fan, and Mark Morgan. 2016. A Transformer Feedback Gm Boosting Technique for Gain Improvement and Noise Reduction in mm-Wave Cascode LNAs. IEEE Transactions on Microwave Theory and Techniques. 64(7): 2080-2090.

[43] Guangyin Feng, Chirn Chye Boon, Fanyi Meng, Xiang Yi, Kaituo Yang, Chenyang Li, and Howard C. Luong. 2017. Pole-converging Intrastage Bandwidth Extension Technique for Wideband Amplifiers. IEEE Journal of SolidState Circuits. 52(3): 769-780.

[44] A. Saberkari, S. Kazemi, V. Shirmohammadli, M. C. Yagoub. 2016. Gm-boosted Flat Gain UWB Low Noise Amplifier with Active Inductor-based Input Matching Network. Integration, the VLSI Journal. 52: 323-333.

[45] G. Gramegna, M. Paparo, P.G. Erratico, P. De Vita. 2001. A sub-1-dB NF 2.3-kV ESD Protected 900-MHz CMOS LNA. IEEE J. Solid State Circuits. 36(7): 1010-1017.
[46] K. V. Reddy, K. Sravani. 2017. Low Power Ultra Wide-band Balun LNA using Noise Cancellation and Current-reuse Techniques. Microelectron. J. 61: 114-122.

[47] A. Sahafi, J. Sobhi, Z. D. Koozehkanani. 2016. Linearity Improvement of gm-boosted Common Gate LNA: Analysis to Design. Microelectron. J. 56: 156-162.

[48] S. Arshad, R. Ramzan, K. Muhammad, Q. U. Wahab. 2015. A sub-10mW, Noise Cancelling, Wideband LNA for UWB Applications. AEU-International Journal of Electronics and Communications. 69(1): 109-118.

[49] A. Taibi, M. Trabelsi, A. Slimane, A. A. Saadi, M. T. Belaroussi. 2017. Efficient UWB Low Noise Amplifier with High Out of Band Interference Cancellation, IET Microw. Antennas Propag. 11(1): 98-105.

[50] M. Sun, N. Ning, J. Li, Q. Yu, Z. Shi, Z. Liu, Y. Liu. 2016. 3-10 $\mathrm{GHz}$ Ultra-wideband LNA with Continuously Variable Gain for Wireless Communication. Microw. Opt. Technol. Lett. 58(7): 1697-1699.

[51] Benqing Guo, Jun Chen, Lei Li, Haiyan Jin, Guoning Yang. 2017. A Wideband Noise-canceling CMOS LNA with Enhanced Linearity by using Complementary nMOS and PMOS Configurations. IEEE J. Solid State Circ. 52(5): January 2017.

[52] Mahdi Parvizi, Karim Allidina, Mourad N. El-Gamal. 2016. An Ultra-low-power Wideband Inductorless CMOS LNA with Tunable Active Shunt-feedback. IEEE Trans. Microw. Theor. Tech. 64(6): June 2016.

[53] Taeyoung Chung, Hankyu Lee, Daechul Jeong, Jehyung Yoon, Bumman Kim. 2015. A Wideband CMOS Noisecanceling Low-noise Amplifier with High Linearity, IEEE Microw. Wireless Compon. Lett. 25(8): August 2015.

[54] Hankyu Lee, Taeyoung Chung, Heesong Seo, Inyoung Choi, Bumman Kim, 2015. A Wideband Differential Lownoise-amplifier with IM3 Harmonics and Noise Canceling IEEE Microw. Wireless Compon. Lett. 25(1): January 2015.

[55] Lanqi Liu, Zhaojing Lu, Kefeng Zhang, Zhixiong Ren, Ang Hu, Xuecheng Zou. 2016. Wideband balun-LNA Exploiting Noise Cancellation and $\mathrm{gm}$ Compensation Technique. Electron. Lett. 52(8): 673674. 